\title{
COMBINING LOCAL FEATURES AND PROGRESSIVE SUPPORT VECTOR MACHINE FOR URBAN CHANGE DETECTION OF VHR IMAGES
}

\author{
Chunlei Huo ${ }^{1}$, Bin Fan $^{1}$, Chunhong Pan ${ }^{1}$, Zhixin Zhou ${ }^{2}$ \\ 1. National Laboratory of Pattern Recognition, Institute of Automation, Chinese Academy of Sciences, Beijing, China, 100190. \\ 2. Beijing Institute of Remote Sensing, Beijing, China, 100854.
}

Commission VII/5

KEY WORDS: change detection, local features, change blindness, cognitive mechanisms, progressive transductive SVM

\begin{abstract}
:
The difficulties about change detection of VHR images are analyzed from different perspectives. Motivated by perception and cognition mechanism of human vision, visual change detection principles are discussed, and a unified change detection framework is proposed. To address the difficulties in change detection of VHR images, a novel approach is presented within the framework, which exploits the combination of local features and change vector displacement field to represent the complex changes of VHR images and utilizes transductive SVM(Support Vector Machine) to classify change features progressively. Experiments demonstrate the effectiveness of the proposed approach.
\end{abstract}

\section{INTRODUCTION}

"Everything changes but changes itself"(Kennedy). With the globalization and geographic expansion of human activities, understanding changes becomes increasingly important. Change detection is the apprehension of changes in the world around us. Remote sensing image change detection aims at detecting changes by comparing multiple images of the same scene taken at different times, and it is a powerful tool that can be used in a diverse range of applications such as disaster management, ecosystem monitoring, military surveillance, and so on. With the development of VHR (Very High Resolution) satellites, change detection receives more extensive attention since it can detect the changes at the more detailed spatial scale. However, compared with low-to-moderate resolution remote sensing images, change detection of VHR images is more challenging since the basic premise of change detection(Singh, 1989)(i.e., changes in land cover must result in changes in radiance values and changes in radiance due to land cover change must be large with respect to radiance changes caused by other factors) is broken by the complexities of such data. In detail, the difficulties lie in the following factors:

First, the difficulties lie in the intrinsic complexity of VHR image. The employment of sensors with the improved spatial resolution simplifies the problem of mixed pixels, however, the internal variability within homogenous land-cover classes increases. At the same time, the increased internal variability decreases the statistical separability between different land-cover classes in the spectral data space. The resulting high internal variability and low spectral separability lead to the reduction of the statistical separability between the changed class and the unchanged class. For this reason, traditional change detection approaches(Coppin et al., 2004, Lu et al., 2004, Radke et al., 2005) are difficult to be applied to VHR images without considering the complexities of such data. For the same reason, some key techniques such as image segmentation and image classification are not mature for VHR images, which hamper the digital change detection techniques.

Second, the difficulties lie in the incomprehensive understanding of human visual change detection mechanism. The ability to detect change is important in much of our everyday life. In spite of the pervasiveness of change detection in our lives, it has proven surprisingly difficult to study, and only recently have various approaches begun to converge in terms of what it is and how it is carried out(Rensink, 2002, Simons and Rensink, 2005). The effective change detection algorithms can be designed by imitating recognition principles of human beings, and the computer is only a tool to accelerate the computation. The incomprehensive understanding of visual change detection mechanism hinders the development of change detection techniques.

Last but not least, the difficulties lie in the "human-machine" gap. The gap is caused by the difference between 3-d real world and 2-d digital images, the difference between human eyes and satellite sensors, the difference between human brains and computers. The other cause is the ambiguous definition of "change", i.e., the definition of change is application-specific, task-specific and user-specific(Paul and Alessandro, 2000). The "human-machine" gap makes the change detection an ill-posed problem, and it is more troublesome to be solved by the computers.

Despite the importance of the existing review papers for developing new change detection approaches, it is difficult to analyze the change detection techniques within a general framework. For this reason, a unified change detection framework is presented, from which most of the existing approaches can be generated. With the help of the proposed framework, a novel approach is presented to overcome the above difficulties encountered in urban change detection of VHR images. Compared to the related work, the contributions of this paper lie in the investigation of change detection framework based on visual change detection principles, as well as the derived approach to address the above difficulties.

The paper is organized in five sections. Section 2 describes the proposed framework. Section 3 presents a detailed description of the proposed approach step by step. Section 4 reports the experimental results obtained on real QuickBird images. Finally, section 5 draws the conclusions.

\section{THE UNIFIED CHANGE DETECTION FRAMEWORK}

The disability to detect changes visually is called change blindness, recent research(Simons and Rensink, 2005) indicates change 
blindness cannot be avoid unless the following three requirements are met simultaneously:

1) The objects being observed must be encoded, and the encoded features must be kept in mind.

2) The features encoded before and after changes occur must be compared.

3) The feature difference must be recognized by the observers.

The above change blindness principles indicate that a digital change detection algorithm should consist of at least three parts: feature space, distance space and search space. For digital change detection techniques implemented by the computer, the procedure to simulate human vision system and cognitive mechanisms to search the changes in a computable manner is very necessary. In consequence, an effective digital change detection algorithm can be divided into the following four components:

(1) Feature space, F.

The feature space determines the place where the co-registered images will be compared.

(2) Distance space, D.

The distance space provides the way how the difference between images is measured quantitatively. The combination of the feature space and the distance space is the change feature space.

(3) Search space, S and object function, f.

Given the feature space and the distance space, the search space decides the change maps by the object function. The object function provides the link between the change features and the real change detection result (which cannot usually be observed) or the approximated optimal change detection result, and it can be designed based on certain criterions such as the minimum error rate and the minimum risk.

(4) Search strategy O.

There may be many solutions that can minimize the object function, among which the optimal solution can be achieved efficiently by the search strategy.

For co-registered images $I_{1}$ and $I_{2}$, the change detection problem can then be formulated as the following unified framework:

$$
\arg \min _{s \in S} f\left(D\left(F\left(I_{1}\right), F\left(I_{2}\right), s\right)\right) .
$$

The selection of each component is determined by the types of images to be compared. For example, spectral and structural features can be used in the feature space, and these features can be extracted in pixel- or region-based manner based on the image resolution (low-to-moderate resolution or high resolution). Based on the types of the sensors (SAR or the optical sensor), the distance can be described by difference-based or ratio-based approach.

The proposed change detection framework has the following advantages:

(1)It captures human visual change detection principles as well as the difference between visual change detection and digital change detection.

(2)It is powerful in understanding and analyzing the existing approaches. Most of the existing change detection methods(Coppin

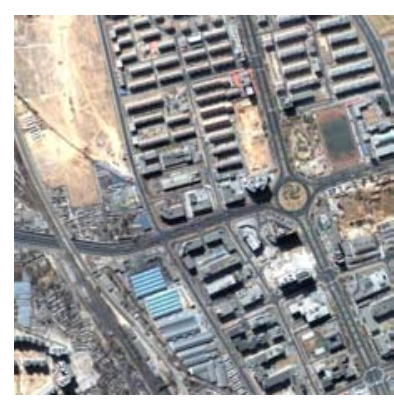

(a)

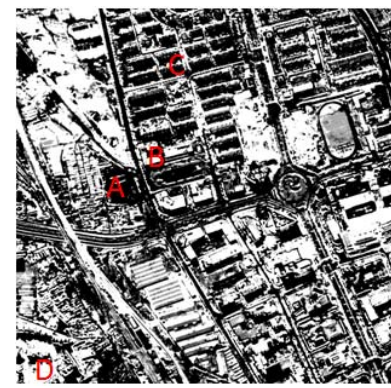

(c)

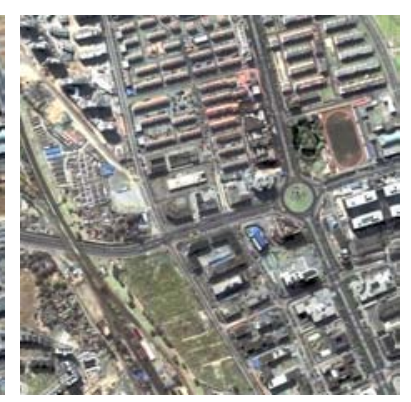

(b)

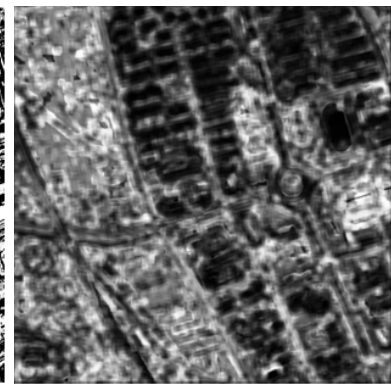

(d)
Figure 1: Illustration of difficulties in change detection of VHR images. (a) and (b):a multi-temporal image pair. (c)change features based on the pixel-wise difference. (d)change features based on the SIFT descriptor difference.

et al., 2004, Lu et al., 2004, Radke et al., 2005) can be included by this framework.

The aim of this paper is to design a new desirable change detection approach for VHR images within the above framework.

\section{THE PROPOSED ALGORITHM}

The focus of this paper is urban change detection of VHR images. For such data, besides the common difficulties of VHR images, the complex morphology caused by human activities must be taken into consideration. A pair of VHR images is shown in Fig.1, some significant changes can be detected with ease by human visual comparison, but some subtle changes are difficult to be detected even by the experienced experts after repeated comparisons. This indicates that the ability of human visual change detection had been overestimated. As shown by the region B, $\mathrm{C}$ and D in Fig.1(c), many false changes are caused by Sun angle variation, shadow and seasonal change, human vision system is robust to such changes, however, it is very hard for the computers. The rationale of the proposed approach is to reduce the missed alarms based on change blindness principles and reduce false alarms by simulating human vision system. In detail, within the proposed framework, we aim to tackle the difficulties of VHR image change detection from the viewpoint of cognitive psychology, i.e., to extracting discriminative local features to represent the complex objects, to measure the difference between objects by the robust metric which takes local nonlinear displacement into consideration, to classify the change features in a progressive fashion. Below we elaborate each component step by step.

\subsection{Feature space}

As stated above, the pure usage of spectral features is too simple for complex urban areas. Take the region A in Fig.1(c) as an example, the structural changes can be detected by human vision, but the compute is "bind" to such changes. In this paper, 


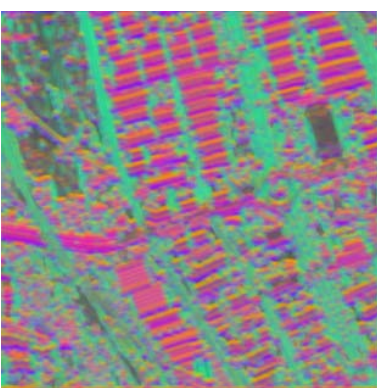

(a)

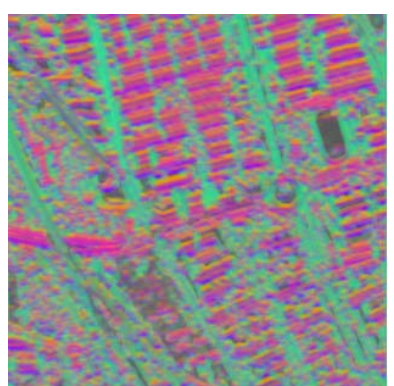

(b)

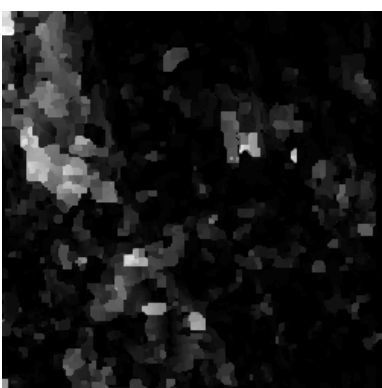

(a)

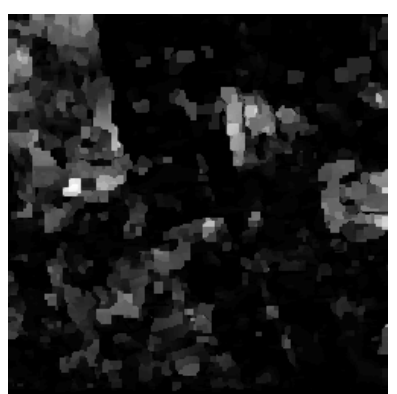

(b)
Figure 2: SIFT feature space for VHR images. (a)SIFT feature for Fig. 1(a). (b) SIFT feature for Fig. 1(b).

SIFT descriptor(Lowe, 2004), i.e., HOG(Histogram of Oriented Gradients), is used as our feature space. In other words, SIFT descriptor is extracted at each pixel to characterize local image structures and encode the contextual information. SIFT is a local descriptor to characterize local gradient information. In (Lowe, 2004), SIFT descriptor is a sparse feature representation that consists of both feature detection and description. In this paper, however, we only use the feature description component. For every pixel in an image, we divide its neighborhood (e.g. $16 \times 16)$ into a $4 \times 4$ cell array, quantize the orientation into 8 bins in each cell, and obtain a 128-d vector as the SIFT representation for a pixel. We call this per-pixel SIFT descriptor SIFT image. Compared to the raw spectral features, SIFT descriptor is a higher level feature and more powerful in capturing the salient structures of man-made objects, this is can be induced from Fig.2 (a) and (b), where the top 3 components of SIFT descriptor after PCA transformation are shown visually. Despite the discriminability of SIFT descriptor, as shown in Fig.1 (d), the algebra difference between SIFT descriptors is too simple to represent the complex changes in VHR images, so the next task is to design a robust distance metric to measure the difference between discriminative local features.

\subsection{Distance space}

Recently, SIFT has been widely used in image matching and object recognition, and most of the literatures compare SIFT descriptors in Euclidean distance space(e.g., the ratio of the nearest neighbors). However, such pixel-wise comparison is not suitable for VHR images. Even if the multi-temporal images are coregistered, local small displacements caused by Sun angle variation or the registration error are inevitable and difficult to remove. In consequence, a robust distance metric is needed which considers the local displacement adaptively to each pixel.

For the same objects taken under different Sun angles, human beings can make the correct decision that no changes happen. The underlying reason is that the appearances of the objects under different Sun angles are regarded to be very similar by human vision system as long as the local displacement is within certain ranges. Based on this observation, for a pixel $p$, the local displacement can be computed by searching the smallest distance within a neighborhood that makes the similarities of SIFT descriptors between the regions around it and the shifted ones in the other images maximized. Given the local displacement specific to each pixel, the distance can be re-computed more accurately by their SIFT descriptors with known displacement. The alternative choice is to represent the difference between two SIFT descriptors by the displacement directly. For a co-registered image pair, if there is no changes happened at the pixel $p$ and its neighbors, their SIFT descriptors should be very close, and the local displacements computed by the above principle should be
Figure 3: Illustration of the distance space presented in this paper. (a)change vector field in horizontal direction. (b)change vector field in vertical direction.

small. And the reverse is also true, large local displacement means changes of high probability. In fact, the latter choice is simpler and is adopted in this paper.

Now, we describe the proposed distance metric in detail. Let $w(p)=(u(p), v(p))$ be the local displacement at $p, s_{i}(p)$ the SIFT descriptor extracted form $I_{i}$ at $p$, the problem of comparing $s_{1}(p)$ and $s_{2}(p)$ is converted to find $w(p)$ which makes the following equation minimized:

$$
\begin{array}{r}
E(w)=\sum_{p}\left(\left\|s_{1}(p)-s_{2}(p+w(p))\right\|_{1}, t\right)+ \\
\sum_{p} \phi(|u(p)|+|v(p)|)+ \\
\sum_{(p, q) \in \mathcal{N}}(\alpha(|u(p)-u(q)|, d)+(\alpha(|v(p)-v(q)|, d)))
\end{array}
$$

The above equation contains a data term, small displacement term and smoothness term (i.e., spatial regularization). The data term in Eqn. (2) constrains the SIFT descriptors to be compared along with the displacement $w(p)$. The small displacement term in Eqn. (3) constrains the displacement vectors to be as small as possible when no other information is available. The smoothness term in Eqn. (4) constrains the displacement vectors of adjacent pixels to be similar. $t$ and $d$ are the thresholds. In order to reduce the complexity by distance transform function(Felzenszwalb and Huttenlocher, 2006) and achieve better convergence by sequential belief propagation (BP-S) (Szeliski et al., 2008), in the objective function, truncated L1 norms are used in both the data term and the smoothness term.

Fig.3(a) and Fig.3(b) show the horizontal and vertical displacements visually. Compared to Fig.1(c) and Fig.1(d), the change information contained in Fig.3(a) and Fig.3(b) is more discriminative. After achieving $w(p)$, the probability of changes at $p$ can be represented by the magnitude and orientation of $w$. The next step is to classify the above 2-d change feature to distinguish the changed class from the unchanged class.

\subsection{Search space}

To design a desirable search space, it is necessary to analyze the cause and characteristic of the local nonlinear displacement. The local displacement is mainly caused by the real changes or the false changes caused by the impacts such as Sun angle variation and the registration error. In detail, the magnitudes of displacement vector corresponding to real changes are large, and the orientations of displacement vector are out of order. In contrast, the magnitudes of displacement vector corresponding to the unchanged class are small, the orientations of displacement vector 
caused by Sun angle variation are of the same direction. Different types of changes are visually shown in Fig.4(a), where C,U and $\mathrm{F}$ denotes the changed class, the unchanged class and false changes caused by Sun angle variation respectively. $T$ are change features to be classified. In fact, the displacement orientation caused by Sun angle variation is closely related to the orientation of shadow. As shown in Fig. 5, for the images after being rectified, the direction of local displacement caused by Sun angle variation is vertical(or with slight variation). In other words, if the displacements are large in both horizontal and vertical directions, the probability of the corresponding pixel is changed is very high; if the displacement is large in only horizontal or vertical direction, the probability of changes caused by Sun angle variation is very high. For a pixel $p$, the corresponding change vector displacement $w(p)=[u(p), v(p)]$ and the magnitude $m(p)=\sqrt{u(p)^{2}+u(p)^{2}}$, we define the above three change types as follows: $C=\left\{p \mid m(p) \geq \tau_{1}, s(p) \geq \tau_{4}\right\}$, $U=\left\{p \mid m(p) \leq \tau_{2}\right\}, F=\left\{p \mid m(p) \leq \tau_{3}, s(p)<\tau_{4}\right\} . s(p)$ is to represent the degree and the direction(along the horizontal and/or vertical direction(s)) of changes . Motivated by Harris corner detector techniques, $s(p)=u(p) * v(p)-k *(u(p)+v(p))^{2}$ is used in this paper, $\mathrm{k}=0.04$. Since the changes $F$ is not the real changes of interest, we add it to the unchanged class, i.e., $U=\left\{p \mid m(p) \leq \tau_{2}\right\} \cup\left\{p \mid m(p) \leq \tau_{3}, s(p)<\tau_{4}\right\}$.

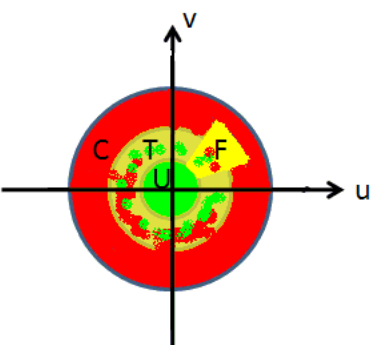

(a)

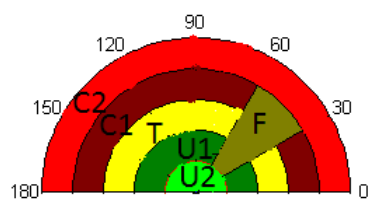

(b)
Figure 4: Different characteristics with respect to different types of changes. (c)distributions of change features in Euclidean coordinates. (d)distributions of change features in polar coordinates.

The task of search space is thus to determine $\tau_{1}-\tau_{4}$ and discriminate $\mathrm{C}$ from $\mathrm{U}$. As shown in Fig.4(b), the representation of change vector field in polar coordinate is more convenient for computation. The objective function is to make the distance between the changed class and the unchanged class maximized. Considering the complex statistical distributions of the changed class and the unchanged class, SVM(Support Vector Machine), a distributionfree classifier, is used to classify the object-specific change features. However, the traditional SVM needs training samples labeled beforehand, and the manual labeling fashion is not practical for real applications. In this paper, this problem is addressed by the progressive transductive SVM, which is implemented by the following search strategy.

\subsection{Search strategy}

The search strategy used in this paper is composed of two steps: initial classification and refined classification. In initial classification, some potential training samples are selected automatically(e.g., the regions $C_{2}, U_{2}$ and $F$ in Fig.4(b)), and the initial change map is determined by the initial separating hyperplane. In refined procedure, the performance is improved gradually by adjusting the training samples(e.g., the regions $C_{1}, U_{1}$ and $T$ in Fig.4(b)) dynamically.

3.4.1 Initial Classification The key to automatic training sample selection is to determine $\tau_{i}(i=1, \ldots, 4), \tau_{1}-\tau_{3}$ are related to $m(p)$, and $\tau_{4}$ is related to $s(p)$. Since the training samples will be tuned iteratively in the latter procedure, a heuristic approach is used to determine $\tau_{i}$ approximately, i.e., let $\tau_{i}(i=1, \ldots, 3)$ be the $\alpha_{i} \times N$ largest number of $m(p), \alpha_{i}$ reflects the fraction of the changed/unchanged class, $N$ is the total number of features. In this paper, $\alpha_{i}=0.8, \alpha_{2}=0.2, \alpha_{3}=0.5, \tau_{4}=50$. By this way, the training set $S_{\text {train }}=S^{c} \bigcup S^{u}$ can be achieved, $S^{c}=\left\{\left(x_{p}, 1\right)\right\}$ and $S^{u}=\left\{\left(x_{p},-1\right)\right\}, x_{p}$ is the 2-d change feature, $x_{p}=[m(p), s(p)]$. The initial classifier is then obtained by the inductive SVM, and the initial change map can be achieved by computing the decision function values on all unlabeled examples.

Due to the uncertainty of training samples and the inherent nature of inductive SVM, the initial change map may not be accurate enough. The uncertainty of training samples lies in the fact that the initial training samples are selected based on the approximated thresholds and limited to represent the whole change feature set. The inherent nature of inductive SVM lies in the purpose to optimize the classification performance over all possible future test data, In fact, this is not necessary since we are only interesting in the features extracted from the images being considered. Consequently, the classification accuracy is refined by the progressive classification, which is implemented by the iterative transductive SVM.

3.4.2 Refined Classification Given a set of independent labeled examples $\left(\mathbf{x}_{1}, y_{1}\right), \ldots,\left(\mathbf{x}_{n}, y_{n}\right), y_{i} \in\{-1,+1\}$, and unlabeled examples $\mathbf{x}_{1}^{*}, \ldots, \mathbf{x}_{k}^{*}$ from the same distribution, the aim of transductive SVM(Collobert et al., 2006) is to minimize the following equation over $\left(y_{1}^{*}, \ldots, y_{k}^{*}, w, b, \xi_{1}, \ldots, \xi_{n}, \xi_{1}^{*}, \ldots, \xi_{k}^{*}\right)$ :

$$
\begin{array}{r}
\min \left(\frac{1}{2}\|w\|^{2}+C \sum_{i=1}^{n} \xi_{i}+C^{*} \sum_{j=1}^{k} \xi_{j}^{*}\right) \\
\text { s.t. } \forall_{i=1}^{n}: y_{i}\left(w \cdot \phi\left(\mathbf{x}_{i}\right)+b\right) \leq 1-\xi_{i}, \xi_{i} \geq 0 . \\
\forall_{j=1}^{k}: y_{j}^{*}\left(w \cdot \phi\left(\mathbf{x}_{j}^{*}\right)+b\right) \leq 1-\xi_{j}^{*}, \xi_{j}^{*} \geq 0 .
\end{array}
$$

Where the regularization parameters $C$ and $C^{*}$ control the generalization capabilities, $\xi_{i}$ and $\xi_{j}^{*}$ are positive slack variables enabling to deal with the permitted errors, $\phi$ is the mapping function. For the change detection algorithm based on the iterative transductive SVM, at each iteration, the non-representative training examples are removed from the training set, and the new representative examples are added from the unlabeled examples to the training set. Based on the hyperplane

$$
\begin{aligned}
& f\left(\mathbf{x}_{i}\right)=\sum_{j} \alpha_{j} y_{j} K\left(\mathbf{x}_{j}, \mathbf{x}_{i}\right)+b \\
= & \sum_{j} \alpha_{j} y_{j}<\phi\left(\mathbf{x}_{j}\right), \phi\left(\mathbf{x}_{i}\right)>+b
\end{aligned}
$$

and Karush-Kuhn-Tucker condition, the training examples $\mathbf{x}_{i}$ can be partitioned into three different categories according to $g_{i}=$ $y_{i} f\left(\mathbf{x}_{i}\right)-1$ (Cauwenberghs and Poggio, 2000): the set $S$ of margin support vectors strictly on the $\operatorname{margin}\left(g_{i}=0\right)$, the set $\mathrm{E}$ of error support vectors exceeding the $\operatorname{margin}\left(g_{i}<0\right)$, and the remaining set $\mathrm{R}$ of reserve vectors exceeding the $\operatorname{margin}\left(g_{i}>0\right)$. At the next iteration, the set $\mathrm{E}$ should be deleted from the training set since its label is inconsistent with the current separating hyperplane, and it contradicts the assumption that the objective function has been minimized. For the unlabeled examples $\mathbf{x}_{1}^{*}, \ldots, \mathbf{x}_{k}^{*}$, only those lying within the margin band are important for the later classification since the adding of them to the training set may change the separating hyperplane. To keep the whole classification stable, at each iteration, we add the new representative training examples in a pair-wise manner(the number 


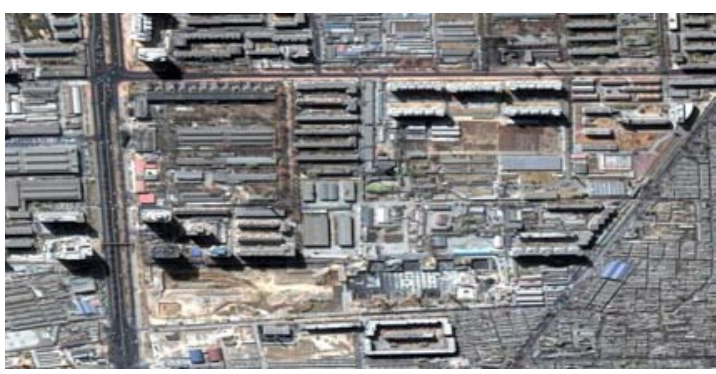

(a)

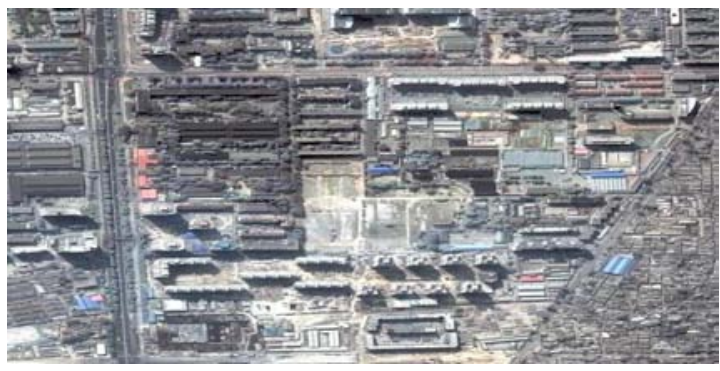

(b)

Figure 5: Images used in this paper. (a)Image taken in 2002. (c)Image taken in 2003.

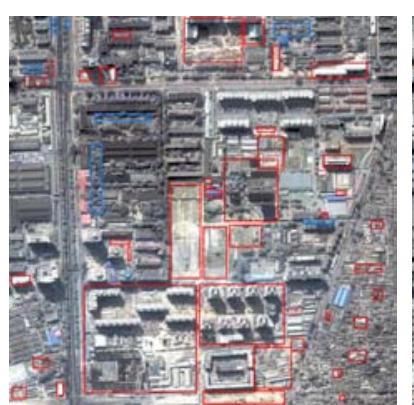

(a)

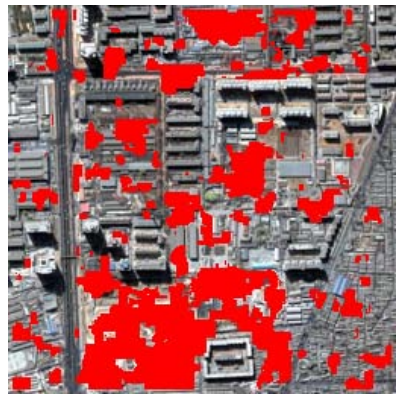

(b)
Figure 6: Results comparison. (a)reference change map. (b)change map by the proposed approach.

of the representative positive examples is equal to the number of the representative negative examples) to the training set whenever possible. However, if there is no such pair, only representative positive or negative examples are picked and added to the training set. In this context, the "representative" examples are the ones that lie within the margin band and are closest to the margin. The iteration continues unless all the unlabeled examples are outside the margin band. By this way, the accuracy is improved iteratively by dynamically selecting the "representative" training samples and gradually tuning the separating hyperplane.

\section{EXPERIMENTAL RESULTS AND DISCUSSION}

To assess the effectiveness of the proposed approach, many experiments were conducted on different datasets. For space limitation, only the results of one dataset are shown in this paper. The images used in this paper are shown in Fig. 5, which are taken by QuickBird satellite over Beijing in 2002 and 2003. The images are composed of three channels (red, green and blue) and the image resolution is $60 \mathrm{~cm} / \mathrm{pixel}$. The size of the images is $1024 \times 1024$ pixels. The selected test sites contain complex urban scene with dense buildings.

Fig.6 shows the reference change map and the result by the proposed approach. Due to the complexities and difficulties of VHR images, the traditional pixel-based evaluation measure is not very helpful for the practical applications. In this paper, we use regionbased false alarms and missed alarms to evaluate the proposed approach in a more objective manner. As seen from Fig. 6(a), the total number of changed regions is 65,8 regions are caused by vegetation change(such changes are labeled in blue), and 57 regions are related to building changes(this type of changes is labeled in red). By comparing Fig. 6(a) and Fig. 6(b), most changes are detected correctly by the proposed approach. After careful validation by the experienced experts, the performances of the proposed approach are summarized as follows:

1) Most building changes are detected correctly with high confidence. As shown in Fig. 7, the structures of buildings as well as the corresponding changes are very complex and difficult to be captured by the traditional approach, however, the proposed approach can detect such complex changes correctly. Moreover, as shown in the right-bottom part of Fig.6(b), some subtle changes of small buildings are detected unbelievably by the proposed approach. In fact, detecting such small-size subtle changes is very hard even for human vision system. Considering the difficulties of VHR images change detection and the limitations of the existed approaches, such result is very encouraging. Due to the powerful ability of SIFT descriptor, the missed alarms is near to 0 . However, as illustrated by Fig.7(d)-(f), one shortage of the proposed approach is that some changed regions are detected partially, the reason lies in the limitation of SIFT descriptor in describing the regions of less textures. In detail, the two regions in red rectangles of Fig. 7(d) are not rich in texture, and they are similar in local pattern. In consequence, they are similar in SIFT descriptor, and the change vector displacements are small. Fortunately, this shortage can be tolerated by the experts since the missed partial region can be observed with the guidance of the detected partial region(e.g., yellow circle in Fig.7(f)). If possible, we will use more discriminative and robust feature descriptors in future work.

2) Another challenge of VHR image change detection is the difficulty to remove the false alarms. As illustrated by Fig. 2(a), false alarms are very high by the traditional difference-based approach. In contrast, false alarms are reduced significantly by the proposed approach. As shown in Fig. 7(g)-(i), the proposed approach is very robust to Sun angle variation or the image registration error. The improvement lies in the robust distance metric represented by change vector displacements. In addition, it is also attributed to the training sample selection strategy. The initial training samples selected by the proposed approach is shown in Fig.8(a), where the changed samples and the unchanged samples are shown in red and green respectively, Fig.8(b) shows the distribution of the change features in the polar coordinate, the blue indicates the unlabeled features to be classified. The reasonability of training sample selection principle can be induced from Fig.8(a), where some changed building are chosen as the changed samples, and some false changes are chosen as the unchanged samples. The features shown in blue in Fig. 8(b) lie within the border between the changed and unchanged regions, they are classified after progressive tune of SVM hyperplane and multiple switches of training sample labels. If no additional considerations are added in determining the initial unchanged training samples, such improvements cannot achieved. Nevertheless, as shown in Fig.7(j)-(1), due to the impacts caused by the vehicle, vegetation and image quality, false alarms are still inevitable. There are about 6 false alarm regions. Compared to the high false alarms by the traditional approach, such performance is still compatible.

The above comparisons indicate the effectiveness of the proposed approach. Of course, this effectiveness is closely related to the 


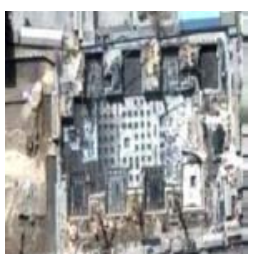

(a)

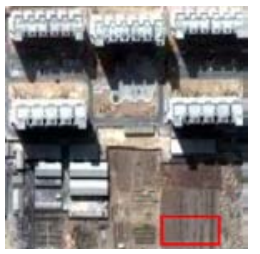

(d)

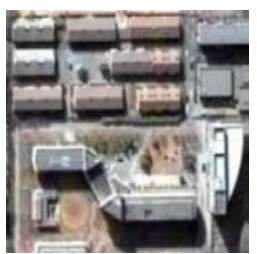

(g)

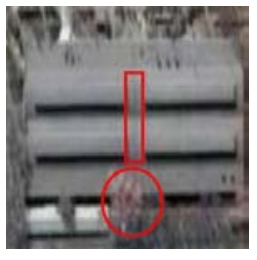

(j)

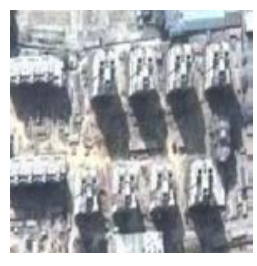

(b)

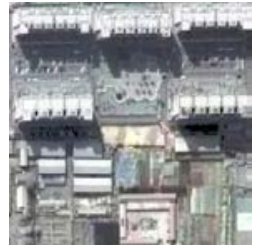

(e)

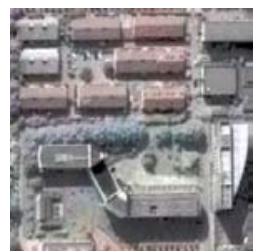

(h)

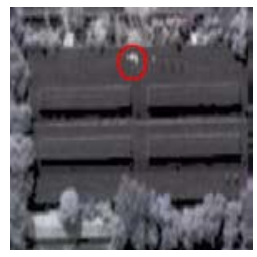

(k)

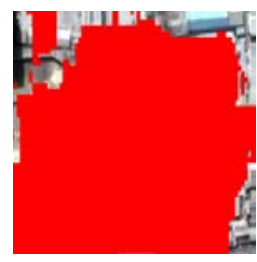

(c)

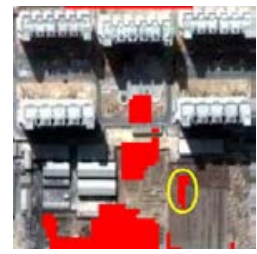

(f)

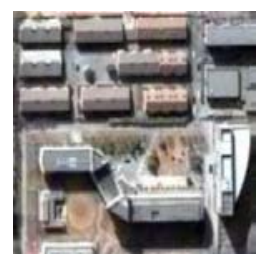

(i)

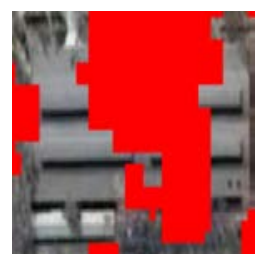

(1)
Figure 7: Four cases used for performance analysis. Case 1(the first row):complex changes are detected correctly. Case 2(the second row): changed regions are detected in part. Case 3(the third row): unchanged regions are detected correctly. Case 4(the fourth row): false alarms.

combination of feature space, distance space, search space and search strategy.

\section{CONCLUSION}

A unified change detection framework is proposed in this paper, which captures the visual change detection principles and is important for analyzing the existing approaches. With the help of the proposed framework, a novel approach is presented for urban change detection of VHR images based on local feature and progressive classification. The contribution of the proposed approach lies in the following three aspects: 1) The change feature is encoded by SIFT descriptor and the nonlinear local displacement, it is discriminative to represent the complex changes and robust to Sun angle variation. 2) The characteristics of different change types are analyzed, based on which the training samples can be selected automatically. 3) A progressive transductive classifier is proposed to classify the change features, it is superior to the traditional approaches by considering the uncertainty of initial training samples and tuning the training samples as well as the hyperplane simultaneously. The proposed approach has advantages over the related techniques in accuracy, efficiency, robustness and automation.

Despite of the promising results, many future developments need be considered in the future work. For example, to make the pro-

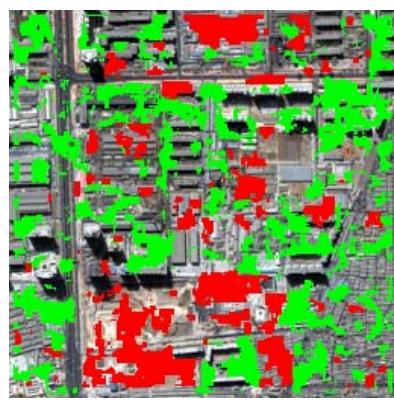

(a)

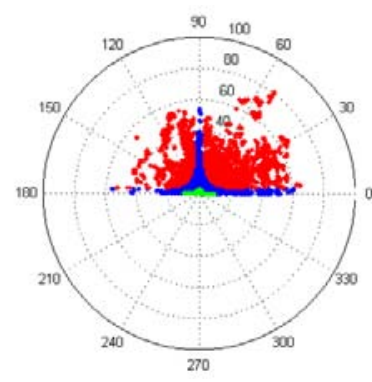

(b)
Figure 8: Automatic sample selection. (a)initial training samples selected by the proposed approach. (b)visualization of training samples in polar coordinate.

posed approach more effective, more discriminative features need being studied in depth.

\section{ACKNOWLEDGEMENTS}

This work was supported by Natural Science Foundation of China under Grant No. 61005013, 60873161, 60723005.

\section{REFERENCES}

Cauwenberghs, G. and Poggio, T., 2000. Incremental and decremental support vector machine learning. Advance in Neural Information Processing Systems.

Collobert, R., Sinz, F. and Weston, J., 2006. Large scale transductive svms. Journal of Machine Learning Research 7, pp. 16871712.

Coppin, P., Lambin, E. and Jonckheere, I., 2004. Digital change detection methods in ecosystem monitoring: a review. Int. Journal of Remote Sensing 25(9), pp. 1565-1596.

Felzenszwalb, P. F. and Huttenlocher, D. P., 2006. Efficient belief propagation for early vision. Int. J. Computer Vision 70(1), pp. 41-54.

Lowe, D. G., 2004. Distinctive image features from scale invariant keypoints. Int. J. Computer Vision 60(2), pp. 91-110.

Lu, D., Mausel, P. and Brondizio, E., 2004. Change detection techniques. Int. Journal of Remote Sensing. 25(12), pp. 23652407.

Paul, C. and Alessandro, A., 2000. Toward specification-driven change detection. IEEE Trans. Geoscience and Remote Sensing 38(3), pp. 1484-1488.

Radke, R. J., Andra, S. and Al-Kofahi, O., 2005. Image change detection algorithms: a systematic survey. IEEE Trans. Image Processing 14(3), pp. 294-307.

Rensink, R. A., 2002. Change detection. Annual Review of Psychology 53, pp. 245-277.

Simons, D. J. and Rensink, R. A., 2005. Change blindness: Past,present, and future. Trends in Cognitive Sciences 9, pp. 1620.

Singh, A., 1989. Digital change detection techniques using remotely-sensed data. Int. J. Remote Sensing 10(8), pp. 9891003.

Szeliski, R., Zabih, R. and Scharstein, D., 2008. A comparative study of energy minimization methods for markov random fields with smoothness-based priors. IEEE Transactions on Pattern Analysis and Machine Intelligence 30(6), pp. 1068-1080. 